recognition). Maricq et al. described last centrury, with the widefield technique (magnification $\mathrm{X} 12-14$ ) the scleroderma pattern. This pathognomonic combination contains the following: a striking widening of all three segments of the capillary loop (arterial, venous and intermediate), loss of capillaries, disorganization of the nailfold capillary bed. Many branched "bushy" capillaries may also be observed. In 2000, Cutolo et al. qualitatively assessed the nailfolds of an SSc cohort with patients fulfilling the American College of Rheumatology (ACR) criteria for SSc with the nailfold videocapillaroscopic (NVC) technique (magnification X200). According to the different proportions of the hallmark parameters of the scleroderma pattern (giants, capillary loss, hemorrhages and (neo)angiogenesis Cutolo et al. defined three patterns "early", "active" and "late".

The central role of capillaroscopy in distinction between a primary and secondary RP due to SSc is reflected by the fact that capillaroscopy is one of the new ACR/EULAR criteria for classifying a patient as having SSc.

Besides playing a paramount role in distinguishing a primary from secondary RP, capillaroscopy has an additional role. It can inform the rheumatologist dealing with a patient population with merely the RP and no other signs of a CTD, who will futurely develop SSc. This role is reflected by capillaroscopy playing a central role in the LeRoy and VEDOSS criteria for (very) early diagnosis of SSc.

What about capillaroscopic morphology in connective tissue diseases other than SSc?

No large scale prospective cohorts exist describing capillaroscopic morphology in connective tissue diseases other than SSc. Moreover, several morphologic defintions exist across literature of different schools. The EULAR Study Group on microcirculation in Rheumatic diseases was set up in 2014 to tackle, in between others these working points.

Disclosure of Interest: None declared

DOI: 10.1136/annrheumdis-2017-eular.7161

\section{SP0099 WHY CAPILLAROSCOPY CAN PREDICT DISEASE SEVERITY AND PROGNOSIS}

M. Cutolo on behalf of EULAR Study Group on Microcirculation. Internal Medicine, Research Lab Division Rheumatology University of Genova, genova, Italy

Nailfold capillaroscopy (NVC) is today considered to be safe biomarker in order to make an early diagnosis of slected Connective Tissue Diseases (CTDs) in presence of Raynaud' phenomenon, and to measure progressive microvascular and tissues damage including response to long term treatment,

Systemic sclerosis is the only CTD to date in which prognostic indices have been described to predict clinical complications. Predictions have been made based on baseline capillaroscopic images and based on sequential capillaroscopic follow-up.

Baseline qualitative-assessed scleroderma patterns have been described to be linked with future organ involvement in any of the nine organ systems affected by SSc according to the disease severity scale of Medsger (general, peripheral vascular, skin, joint, muscle, gastrointestinal tract, lung, heart and kidney) (1). Additionally, baseline capillaroscopic evaluations have been linked to future development of digital trophic lesions in SSc. A simple scoring system has been used recently in the largest pan-European study evaluating the role of capillaroscopy in predicting digital ulcers in SSc (2). More specifically, in this study, simply the number of capillaries per linear $\mathrm{mm}$ had been evaluated. Besides counting the number of capillaries/capillary alterations, dimensions can also be measured. The latter has also been used in prediction of patients with RP whether, because of SSc, there will be a possibility for them to develop a secondary RP. Similarly, it has recently been attested that if the average capillary diameter (average of the largest apical, efferent and afferent limb in 16 fields, more specifically 2 fields per finger, fingers $2-5$ from each hand) is less than $30 \mu \mathrm{m}$ in a group of patients with RP but without scleroderma characteristic findings on nailfold videocapillaroscopy (NVC), the patient has a low chance of developing SSc, while if $>30 \mu \mathrm{m}$, then the patient has $50 \%$ chance to develop SSc (3). Concerning the ability of capillaroscopy to measure response to treatment, there are yet no prospective randomized, double-blind, placebo-controlled trials, evaluating the ability of capillaroscopy to monitor response to therapy concerning RP-related outcome measures. It is noteworthy and promising that in small studies showing response of immunosuppressive/vasomodulating treatment on disease severity, outcome measures are available (4-6).

References:

[1] V. Smith et al. J Rheumatol 40,2013,52-7.

[2] M. Cutolo et al. Arthritis Rheumatol 68,2016,2527-39.

[3] A. Trombetta et al. J Rheumatol 43,2016, 599-606.

[4] I. Miniati et al. Ann Rheum Dis 68, 2009;94-8.

[5] V. Smith et al. J Rheumatol 43, 2016,995-6 6.

[6] A. Trombetta, C et al. J Rheumatol. 2016;43:2033-41.

Disclosure of Interest: None declared

DOI: 10.1136/annrheumdis-2017-eular.7295

\section{SP0100 HOW TO SELECT THE MOST APPROPRIATE CAPILLAROSCOPIC DEVICE: PROS AND CONS}

W. Hermann. Department of Rheumatology and Clincial Immunology, Kerckhoff-Klinik, Bad Nauheim, Germany

One of the most important indications for performing capillaroscopy is to differentiate between primary and secondary Raynaud's syndrome. Different kinds of microscopes are at hand and generally vary in terms of picture quality or price. Before purchasing a microscope and capillaroscopy software, several considerations about the required standards of examination should be made; some of which are summarized as follows:

- The region of interest (ROI). Normal capillaries have a mean diameter of about $8 \mu \mathrm{m}$. For an accurate assessment a magnification of 100-200x is recommended, for an overview the magnification of 50x is sufficient.

- Measurement. Beside qualitative measures like changes in vessel architecture, there should be the possibility of quantifying the number of capillaries $/ \mathrm{mm}$ or vessel diameters.

- Documentation. All parts of the examination have to be stored and assigned to patient and case.

- Practical aspects and handling of the device.

- Different kinds of microscopes are on the market of which three will be discussed in detail. Briefly summarized:

- Stereo microscopes.

Advantages: Very good image quality, zooming in and out without problems, relatively easy to use.

Disadvantages: device is not mobile, in patients with finger contractures examinations are difficult to perform, relatively high costs.

- Videocapillaroscopes:

Advantages: Very good image quality, easy to use, "gold standard" for capillaroscopy.

Disadvantages: No overview, zooming in and out not applicable (change of lenses required), relatively high costs.

- USB microscopes:

Advantages: low costs, zooming in and out without problems, easy to use.

Disadvantages: limited picture quality, documentation laborious.

Selecting a capillaroscopic device depends on the conditions of use ("quick look" vs. "academic evaluation and follow up"), which should be clarified before buying a device. The price range is significant and usually differs between $100 €$ for USB microscopes and up to $10,000 €$ for stereo and videocapillaroscopes

Literature:

[1] Cutolo $M$ et al. How to perform and interpret capillaroscopy. Best Pract Res Clin Rheumatol. 2013;27(2):237-48.

[2] Cutolo $M$ et al. State of the art on nailfold capillaroscopy: a reliable diagnostic tool and putative biomarker in rheumatology? Rheumatology (Oxford). 2013;52(11):1933-40.

Disclosure of Interest: None declared

DOI: 10.1136/annrheumdis-2017-eular.7206

\section{THURSDAY, 15 JUNE 2017 \\ Ultrasound, clinical, diagnostic and therapeutic skills I \& II}

\section{SP0101 DIAGNOSTIC AND THERAPEUTIC ULTRASOUND-GUIDED PROCEDURES}

P. Mandl. Rheumatology, Medical University of Vienna, Vienna, Austria

The lecture will provide an overview of sonography-guided musculoskeletal interventions which can be grouped broadly as diagnostic and therapeutic procedures. Primarily diagnostic procedures include arthrocentesis, biopsy from various musculoskeletal tissues (synovial, bone, muscle etc.), aspiration of fluid from cystic lesions, tendon sheaths and bursae. The therapeutic group features joint and soft tissue injections, needling of periarticular calcification, including barbotage. Both indirect and direct-guidance techniques will be detailed and published literature on accuracy, outcome and safety of sonographic-guided interventions will be reviewed.

Disclosure of Interest: None declared

DOI: 10.1136/annrheumdis-2017-eular.7272

\section{SP0102 HOW TO PERFORM A QUICK AND EFFICIENT PHYSICAL EXAMINATION}

M. Doherty. Academic Rheumatology, University of Nottingham, Nottingham, United Kingdom

The GALS (Gait, Arms, Legs, Spine) screen is a quick and reasonably sensitive way to detect common musculoskeletal (MSK) abnormalities as part of a general medical assessment (1). However, for a person with MSK complaints a detailed assessment is required to determine the diagnosis and the impact of the condition on that person. The key starting point is the history. This needs to be holistic and individualised as the enquiry proceeds since the impact of any condition is 